\title{
Exercícios físicos na doença arterial obstrutiva periférica
}

\author{
Physical exercise in peripheral occlusive arterial disease \\ Elenir Carlot Locatelli, Suélen Pelizzari, Kátia Bilhar Scapini, Camila Pereira Leguisamo, \\ Alexandre Bueno da Silva*
}

\section{Resumo}

A maioria dos pacientes portadores de claudicação intermitente, um aspecto clínico da doença arterial periférica, tem importante limitação nas atividades físicas e redução na qualidade de vida. O objetivo deste estudo foi realizar uma revisão da literatura sobre a intervenção através de exercícios em portadores de doença arterial obstrutiva periférica com claudicação intermitente. Trata-se de uma revisão de artigos científicos consultados nos bancos de dados da BIREME, PubMed e SciELO, através das fontes LILACS e MEDLINE e a partir dos descritores em Ciências da Saúde claudicação intermitente, doenças vasculares periféricas, reabilitação, exercício e terapia por exercício. Concluiu-se que, apesar da variabilidade dos regimes de caminhada identificados na literatura, o treino aeróbio, de uma forma geral, proporciona benefícios a pacientes portadores de doença arterial obstrutiva periférica com claudicação intermitente, principalmente na melhora do desempenho de caminhada, o que pode ter impacto significativo na qualidade de vida desses pacientes.

Palavras-chave: Claudicação intermitente, doenças vasculares periféricas, reabilitação, exercício, terapia por exercício.

\section{Introdução}

A doença arterial obstrutiva periférica (DAOP) tem como principal causa a aterosclerose que, de forma evolutiva, provoca obstrução nas artérias ${ }^{1}$. A claudicação intermitente (CI), um espectro clínico da DAOP, caracteriza-se pela ocorrência de dor no paciente ao deambular, que, muitas vezes, restringe as suas atividades diárias ${ }^{2-4}$.

Estima-se que a prevalência da doença arterial periférica se encontra em entre 3 e $10 \%$ da população, aumentando para $20 \%$ quando se trata de pessoas de mais de 70 anos. A incidência da doença é maior (2:1) em homens negros, hispânicos e com fatores de risco para doença aterosclerótica ${ }^{5}$. Os fatores de risco para aterosclerose podem ser divididos em modificáveis (tabagismo, sedentarismo, obesidade, estresse, hiperlipidemia, hipertensão arterial) e não modificáveis (diabetes mellitus, hipertensão familiar,

\begin{abstract}
Most patients with intermittent claudication, a clinical aspect of peripheral arterial disease, have important limitations on physical activity and a reduced quality of life. The purpose of this study was to review literature on exercise intervention for patients with peripheral occlusive arterial disease and intermittent claudication. BIREME, PubMed (MEDLINE), SciELO and LILACS databases were searched for the terms intermittent claudication, peripheral vascular diseases, rehabilitation, exercise, exercise therapy, all of which were taken from the list of Health Science Descriptors (BIREME). It was concluded that, despite the variability of walking regimens identified in the literature, the aerobic training is of general benefit to patients with peripheral arterial disease and intermittent claudication, mainly improving their walking performance, which can have a significant impact on the quality of life of these patients.
\end{abstract}

Keywords: Intermittent claudication, peripheral vascular diseases, rehabilitation, exercise, exercise therapy.

trombofilias, sexo, idade e hereditariedade $)^{2,6-11}$. O tratamento da DAOP inclui modificação dos fatores de risco, mudanças no estilo de vida ${ }^{8,11}$ e o uso de drogas específicas geralmente associado a exercícios físicos, sendo que os medicamentos associados à reabilitação com exercícios reduzem a necessidade de intervenção cirúrgica ou endovascular, limitando os procedimentos invasivos para pacientes que tem piora de seus sintomas s, $^{3,92-15}$.

A doença aterosclerótica está associada a elevada morbidade e mortalidade ${ }^{9,16}$, mas a taxa de mortalidade pode ser reduzida através de atividade física regular ${ }^{17,18}$. A utilização do exercício físico com fins terapêuticos tem sido uma proposta de tratamento para pacientes portadores de $\mathrm{CI}^{5}$. A fisioterapia através de exercícios pode trazer benefícios para esses pacientes, tais como: aumento do desempenho de caminhada ${ }^{18,19-23}$, melhora na qualidade de

\footnotetext{
* Faculdade de Educação Física e Fisioterapia, Universidade de Passo Fundo, Passo Fundo, RS.

Não foram declarados conflitos de interesse associados à publicação deste artigo.

Artigo submetido em 14.02.09, aceito em 01.07.09.

J Vasc Bras. 2009;8(3):247-254.

Copyright $(\odot 2009$ by Sociedade Brasileira de Angiologia e de Cirurgia Vascular
} 
vida e prognóstico da doença ${ }^{24,25}$, redução dos níveis de estresse, aumento do fluxo sanguíne ${ }^{26,27}$, aumento no limiar de dor e melhora da capacidade funcional, facilitando a realização de atividades da vida diária e ocupaciona ${ }^{25}$. A reabilitação com exercícios físicos, além de ser eficaz e não invasiva, também se revela de baixo custo quando comparada com a intervenção cirúrgica ${ }^{28}$, sendo que esta é opção de escolha quando não há resultados satisfatórios através do tratamento conservador e deve ser indicada quando a distância de caminhada do paciente esteja sendo progressivamente afetada ${ }^{29,30}$. Apesar dos vários efeitos benéficos, a intervenção fisioterapêutica através de exercícios não é utilizada de forma rotineira na prática clínica. Talvez isso se deva ao fato de que ainda existem na literatura muitas controvérsias quanto ao programa de exercícios mais adequado para o tratamento da DAOP. Por isso, o objetivo do presente estudo foi realizar uma revisão de estudos sobre a intervenção através de exercícios em pacientes portadores de DAOP com CI.

\section{Método}

Trata-se de uma revisão de literatura, na qual foram consultadas as bases de dados BIREME, LILACS, SciELO e PubMed (MEDLINE) através dos descritores em Ciências da Saúde claudicação intermitente, doenças vasculares periféricas, reabilitação, exercício, terapia por exercício e os termos correspondentes em língua inglesa intermittent claudication, peripheral vascular disease, rehabilitation, exercise, exercise therapy. As buscas foram realizadas restringindo a data para artigos publicados entre 2004 e 2008 em língua inglesa, portuguesa e espanhola, sendo incluídos os estudos que abordavam casos nos quais a intervenção em pacientes com DAOP e CI se dera exclusivamente através de exercício físico. Uma vez realizada a busca, foram excluídos os artigos que tratavam de membro superior, alterações venosas, renais, intervenção ou cointervenção cirúrgica ou medicamentosa. Desta forma, foram analisados 42 artigos dos quais só 12 preencheram os critérios de inclusão deste estudo.

\section{Resultados e discussão}

A restrição da busca a artigos publicados entre 2004 e 2008 e nas línguas inglesa, portuguesa e espanhola, apesar de determinar a exclusão parcial da produção de conhecimento global, provavelmente dê conta das evidências mais atuais e relevantes sobre o assunto em apreço no ce- nário internacional e nacional. A Tabela 1 apresenta os dados dos estudos selecionados, sendo que, do total de estudos (12), apenas quatro $(33,3 \%)$ eram ensaios clínicos controlados $^{20,22,31,32}$. Setecentos e noventa $(\mathrm{n}=790)$ pacientes fizeram parte dos 12 estudos analisados. O número da amostra variou de 11 a 202 pacientes. Em dois estur $\operatorname{dos}^{25,33}$, a amostra incluiu menos de 20 pacientes; a metade dos estudos ${ }^{20,22,27,31,34,35}$ envolveu de 20 a 75 pacientes; em dois estudos ${ }^{32,36}$, a amostra foi de 75 a 100 pacientes; e dois estudos $^{37,38}$ envolveram mais de 100 pacientes. A maioria dos estudos analisados utilizou treinamento físico supervisionado (10 / 83, 3\%). Em relação ao tempo de duração da intervenção através de exercícios, esta variou de 10 semanas $^{38}$ a 1 ano $^{20}$. Cinco estudos ${ }^{22,27,31,33,35}$ tiveram duração de 12 semanas, um estudo ${ }^{25}$ durou 20 semanas e quatro tiveram duração de 24 semanas ${ }^{32,34,36,37}$. Sabe-se que programas de exercícios com duração de 3 meses já trazem benefícios para a vida dos pacientes, como se constatou na maioria dos estudos revisados; contudo, um período de 6 meses ou mais de treinamento é considerado como preditor de resposta a um programa de treinamento para pacientes com $\mathrm{DAOP}^{5}$. No que se refere à modalidade dos exercícios, a maioria (oito, 66\%) dos trabalhos s, $27,28,32,33,35-37^{-1}$ envolvia treino aeróbio como única modalidade de exercício, sendo que a caminhada foi a atividade mais utilizada. A sistemática dos exercícios de caminhada variou de um estudo para outro, incluindo desde caminhada não supervisionada realizada na comunidade ${ }^{20,33}$ até um programa formal de exercício supervisionado envolvendo caminhada em esteira ${ }^{22,25,27,34-38}$. Os procedimentos adotados em cada estudo podem ser visualizados na Tabela 1. Quatro estudos (44\%) combinaram treinamento aeróbio com alguma forma de exercício resistido de baixa intensidade ${ }^{25,31,34,38}$. Nenhum dos estudos utilizou somente exercícios resistidos como forma de intervenção. O treino de caminhada, especialmente o realizado em esteira, tem se mostrado mais eficaz do que o treino de força ou a combinação de modalida$\mathrm{des}^{5}$. No entanto, poucos trabalhos têm se dedicado a avaliar os efeitos do treinamento resistido em portadores de DAOP. De acordo com Câmara et al. ${ }^{39}$, os pacientes acometidos por essa doença podem ser beneficiados pelos efeitos terapêuticos dos exercícios resistidos, sendo recomendada a inclusão dessa modalidade no tratamento da DAOP. Contudo, devido à presença de múltiplas doenças e fatores de risco que apresentam esses pacientes, os autores recomendam a adoção de cuidados especiais na prescrição 
dessa modalidade de exercício para essa população. A frequência de treinamento prescrita foi geralmente de três vezes por semana $a^{20,25,31,33,35,36}$, mas três artigos descreveram a utilização de duas sessões semanais ${ }^{22,32,38}$; em um trabalho, a frequência foi de seis sessões semanais ${ }^{37}$; em apenas um trabalho os exercícios foram realizados diariamente ${ }^{27} \mathrm{e}$ um estudo indicou uma frequência variável de duas a três vezes por semana ${ }^{34}$. A intensidade do treinamento aeróbio foi geralmente de baixa a moderada, progredindo de acordo com a tolerância à dor e com o aumento do condicionamento dos pacientes. Os pacientes foram encorajados a se exercitarem próximo ao nível máximo de dor em três estu$\operatorname{dos}^{34,36,38}$ e em outros três foi utilizada como limiar a claudicação de moderada intensidade ${ }^{20,22,33}$. De acordo com Leng, Fowler e Ernst ${ }^{40}$, melhores resultados são obtidos com um programa de exercícios de caminhada realizado três vezes por semana próximo ao nível máximo de dor. Já o Inter-Society Consensus for the Management of Peripheral Arterial Disease (TASC II) ${ }^{5}$ recomenda que o paciente pare de caminhar quando a dor da claudicação for considerada moderada, pois uma resposta de treinamento "menos ótima" seria alcançada se o paciente parasse no início da claudicação. A velocidade inicial de caminhada na esteira foi relatada em seis trabalhos, sendo que em cinco deles foi utilizada velocidade de $3,2 \mathrm{~km} / \mathrm{h}^{20,22,28,33,38}$. Dois estudos utilizaram treinamento aeróbio de alta intensidade e intervalado. Em um deles, o treinamento aeróbio foi realizado em cicloergômetro, e a intensidade foi definida de acordo com o $\mathrm{VO}_{2}$ pico; a intensidade variou entre 85 e $90 \%$ do $\mathrm{VO}_{2}$, e os pacientes se exercitavam durante 2 minutos, descansando por 1 minuto, até completar o total de 40 minutos $^{32}$. Em outro estudo, realizado por Adams et al..$^{35}$, o treinamento consistia em aproximadamente 6 minutos de caminhada em esteira seguidos de 3 minutos de descanso, totalizando seis intervalos por sessão. De acordo com o TASC II $^{5}$, a caminhada em esteira deve ser realizada a uma velocidade e inclinação que induzam claudicação dentro de 3 a 5 minutos, sendo que o paciente deve interromper a caminhada quando a dor da claudicação é moderada e descansar até a claudicação diminuir, retomando, depois, a caminhada até o desconforto moderado da claudicação retornar. Além disso, a velocidade e a inclinação da esteira devem ser aumentadas quando o paciente for capaz de caminhar por 10 minutos ou mais na carga de trabalho mais baixa sem chegar a sentir dor moderada de claudicação, e, ainda, o aumento na inclinação é recomendado se o paci- ente já consegue caminhar a 3,2 km/h. Em geral, a duração dos treinos aeróbios variou de 20 a 40 minutos, sendo que seis estudos relataram a ocorrência de períodos de descanso durante o treino aeróbio ${ }^{20,22,27,32,33,35}$. Segundo o TASC $\mathrm{II}^{5}$, o ciclo de exercício e descanso deve ser de pelo menos 35 minutos no início do programa e aumentar para $50 \mathrm{mi}$ nutos conforme o paciente for se sentindo confortável com as sessões de exercício, mas sempre evitando fadiga ou desconforto excessivo nas pernas. Vários benefícios foram relatados após o treinamento físico, como pode ser verificado na Tabela 1. Alguns desses benefícios são comentados a seguir.

\section{Melhora no desempenho de caminhada}

Apesar da grande variabilidade nos programas de exercícios utilizados, vários estudos demonstraram melhora significante na distância máxima caminhada pelos pacientes com $\mathrm{CI}^{25,27,31,32,34,36-38}$, sendo relatados aumentos de até $191 \%$ após 24 semanas de treinamento aeróbio associado a exercícios resistidos ${ }^{34}$. Incrementos na distância de surgimento da claudicação também foram verifica$\operatorname{dos}^{31,32,34,36-38}$. Foram relatados ainda aumentos no tempo máximo de caminhada ${ }^{20,22,25,33}$, no tempo de caminhada livre de dor ${ }^{20,33}$, na velocidade máxima durante a caminhada e na velocidade livre de dor ${ }^{37}$. Esses resultados demonstram que um programa de exercícios físicos pode aumentar significativamente a capacidade de caminhar de portadores de CI. Ainda, pacientes claudicantes submetidos a treinamento físico, utilizando sessões de caminhada de $40 \mathrm{mi}$ nutos, quatro vezes por semana, mesmo apresentando agravamento da doença arterial de membros inferiores, caracterizado por novas estenoses proximais em segmentos arteriais previamente poupados, não apresentaram qualquer redução da distância máxima de caminhada em resposta ao desenvolvimento de novas lesões arteriais ${ }^{41}$.

\section{Aumento no consumo máximo de oxigênio}

Cinco estudos, todos utilizando somente treinamento aeróbio, avaliaram o consumo máximo de oxigênio, sendo que, em três deles, houve aumento significativo de consu$\mathrm{mo}^{32,33,36}$. Dois estudos não relataram aumento significativo no $\mathrm{VO}_{2}$, talvez devido à baixa intensidade e curta duração da sessão ${ }^{20,22}$ e também à baixa frequência e ao tempo reduzido de treinamento ${ }^{22}$. 
Tabela 1 - Estudos sobre exercício na doença arterial obstrutiva periférica com claudicação intermitente

\begin{tabular}{|c|c|c|c|c|c|c|c|c|c|}
\hline \multirow[t]{2}{*}{ Autores } & \multirow[t]{2}{*}{$\mathrm{n}$} & \multirow[t]{2}{*}{ Grupo } & \multicolumn{4}{|c|}{ Exercício } & \multicolumn{3}{|c|}{ Resultados } \\
\hline & & & Supervisão & Modalidade & Prescrição & Duração & Variáveis & Mudança & $\mathrm{p}$ \\
\hline \multirow[t]{2}{*}{ Manfredini et al. ${ }^{37}$} & \multirow[t]{2}{*}{126} & $\begin{array}{l}\text { EXE } 1 \\
(n=74)\end{array}$ & não & AER & $\begin{array}{l}\text { duas sessões diárias } \\
\text { de } 10 \text { min de cami- } \\
\text { nhada, seis ve- } \\
\text { zes/sem, de acordo } \\
\text { com a velocidade } \\
\text { máxima assintomá- } \\
\text { tica obtida no teste }\end{array}$ & $24 \mathrm{sem}$ & $\begin{array}{c}\text { G EXE } 1 \\
\text { PAS e PAD } \\
\text { ITB } \\
\text { DIC } \\
\text { DMC } \\
\text { VLD } \\
\text { VM }\end{array}$ & $\begin{array}{l}\text { diminuiu } \\
\text { aumentou } \\
\text { aumentou } \\
\text { aumentou } \\
\text { aumentou } \\
\text { aumentou }\end{array}$ & $\begin{aligned} &<0,05^{\mathrm{a}} \\
&<0,001^{\mathrm{a}} \\
&<0,0001^{\mathrm{a}} \\
&<0,0001^{\mathrm{a}} \\
&<0,0001^{\mathrm{a}} \\
&<0,0001^{\mathrm{a}}\end{aligned}$ \\
\hline & & $\begin{array}{l}\text { EXE } 2 \\
(n=52)\end{array}$ & não & AER & $\begin{array}{l}20 \text { a } 30 \text { min diários } \\
\text { de caminhada, seis } \\
\text { vezes/sem, velocid } \\
\text { ade livre determi- } \\
\text { nada pela tolerân- } \\
\text { cia à dor }\end{array}$ & 24 sem & $\begin{array}{l}\text { G EXE } 2 \\
\text { PAS } \\
\text { PAD } \\
\text { ITB } \\
\text { DIC } \\
\text { DMC } \\
\text { VLD } \\
\text { VM }\end{array}$ & $\begin{array}{c}\text { diminuiu } \\
\text { não } \Delta \\
\text { não } \Delta \\
\text { aumentou } \\
\text { aumentou } \\
\text { aumentou } \\
\text { aumentou }\end{array}$ & $\begin{array}{c}<0,05^{\mathrm{a}} \\
- \\
- \\
<0,0001^{\circ} \\
<0,0001^{\circ} \\
<0,0001^{\circ} \\
<0,0001^{\circ}\end{array}$ \\
\hline Wang et al. ${ }^{33}$ & 11 & $\begin{array}{l}\text { EXE } \\
(n=11)\end{array}$ & $\operatorname{sim}$ & AER & $\begin{array}{l}\text { Caminhada em es- } \\
\text { teira com velocidade } \\
\text { inicial de } 3,2 \mathrm{~km} / \mathrm{h} \\
\text { aumentando confor- } \\
\text { me limiar de dor, } \\
\text { por } 1 \text { hora (incluin- } \\
\text { do períodos de } \\
\text { descanso se necessá- } \\
\text { rio), três vezes/sem }\end{array}$ & $12 \mathrm{sem}$ & $\begin{array}{l}\text { TCLD } \\
\text { TMC } \\
\mathrm{VO}_{2} \\
\mathrm{ITB} \\
\mathrm{PCF}\end{array}$ & $\begin{array}{c}\text { aumentou } \\
\text { aumentou } \\
\text { aumentou } \\
\text { não } \Delta \\
\text { não } \Delta\end{array}$ & $\begin{array}{c}<0,01^{\mathrm{a}} \\
<0,001^{\mathrm{a}} \\
<0,05^{\mathrm{a}} \\
\quad- \\
-\end{array}$ \\
\hline Roberts et al. ${ }^{27}$ & 47 & $\begin{array}{l}\text { EXE } \\
(n=47)\end{array}$ & não & AER & $\begin{array}{l}1 \text { hora de cami- } \\
\text { nhada com perío- } \\
\text { dos de descanso, se } \\
\text { necessário, todos os } \\
\text { dias }\end{array}$ & 12 sem & $\begin{array}{c}\text { DMC } \\
\text { FCr, FCe } \\
\text { DPr e DPdm } \\
\text { ITB } \\
\text { FSr e FSdm } \\
\text { LS } \\
\text { QV }\end{array}$ & $\begin{array}{c}\uparrow 76,2 \% \\
\text { diminuiu } \\
\text { diminuiu } \\
\text { não } \Delta \\
\text { aumentou } \\
\text { não } \Delta \\
\uparrow 22,4 \%\end{array}$ & $\begin{array}{l}<0,001^{\mathrm{a}} \\
<0,001^{\mathrm{a}} \\
<0,001^{\mathrm{a}} \\
\quad- \\
<0,001^{\mathrm{a}} \\
\quad- \\
<0,001^{\mathrm{a}}\end{array}$ \\
\hline \multirow[t]{2}{*}{ Crowther et al. ${ }^{20}$} & \multirow[t]{2}{*}{21} & $\begin{array}{l}\text { EXE } \\
(n=10)\end{array}$ & \multirow[t]{2}{*}{$\operatorname{sim}$} & \multirow[t]{2}{*}{ AER } & \multirow[t]{2}{*}{$\begin{array}{l}\text { Caminhada em es- } \\
\text { teira inicialmente a } \\
3,2 \mathrm{~km} / \mathrm{h} \text {, por } 25 \mathrm{a} \\
40 \text { minutos, com } \\
\text { progressão confor- } \\
\text { me limiar de dor, } \\
\text { com períodos de } \\
\text { descanso, se neces- } \\
\text { sário, três vezes/sem }\end{array}$} & \multirow[t]{2}{*}{1 ano } & $\begin{array}{c}\text { TCLD } \\
\text { TMC } \\
\mathrm{VO}_{2} \\
\mathrm{NAF}\end{array}$ & $\begin{array}{c}\text { aumentou } \\
\text { aumentou } \\
\text { não } \Delta \\
\text { não } \Delta\end{array}$ & $\begin{array}{c}<0,05^{\mathrm{a}} \\
<0,01^{\mathrm{a}} \\
- \\
-\end{array}$ \\
\hline & & $\begin{array}{l}\text { CONT } \\
(\mathrm{n}=11)\end{array}$ & & & & & & & \\
\hline \multirow[t]{2}{*}{ Hodges et al. ${ }^{22}$} & \multirow[t]{2}{*}{28} & $\begin{array}{l}\text { EXE } \\
(n=14)\end{array}$ & $\operatorname{sim}$ & AER & $\begin{array}{l}\text { Caminhada na } \\
\text { esteira a } 3,2 \mathrm{~km} / \mathrm{h} \text { e } \\
75 \% \text { da inclinação } \\
\text { máxima obtida no } \\
\text { teste, com duração } \\
\text { e progressão vari- } \\
\text { ando de acordo } \\
\text { com limiar de } \\
\text { dor, com períodos } \\
\text { de descanso até } \\
\text { completar } 30 \text { min } \\
\text { de caminhada, duas } \\
\text { vezes/sem }\end{array}$ & 12 sem & $\begin{array}{c}\mathrm{VO}_{2} \\
\mathrm{TMC} \\
\mathrm{FCe}\end{array}$ & $\begin{array}{l}\text { não } \Delta \\
\uparrow 91 \% \\
\text { não } \Delta\end{array}$ & - \\
\hline & & $\begin{array}{l}\text { CONT } \\
(\mathrm{n}=14)\end{array}$ & & & & & & & \\
\hline
\end{tabular}


Tabela 1 (cont.) - Estudos sobre exercício na doença arterial obstrutiva periférica com claudicação intermitente

\begin{tabular}{|c|c|c|c|c|c|c|c|c|c|}
\hline \multirow[t]{2}{*}{ Autores } & \multirow[t]{2}{*}{$\mathrm{n}$} & \multirow[t]{2}{*}{ Grupo } & \multicolumn{4}{|c|}{ Exercício } & \multicolumn{3}{|c|}{ Resultados } \\
\hline & & & Supervisão & Modalidade & Prescrição & Duração & Variáveis & Mudança & $\mathrm{p}$ \\
\hline Ratliff et al. ${ }^{38}$ & 202 & $\begin{array}{l}\text { EXE } \\
(n=202)\end{array}$ & $\operatorname{sim}$ & COMBO & $\begin{array}{l}5 \text { min de aquecimen- } \\
\text { to em bicicleta } \\
\text { ergométrica seguidos } \\
\text { de exercícios de } \\
\text { alongamento, exercí- } \\
\text { cios resistidos ( } 10 \\
\text { rep), bicicleta ergo- } \\
\text { métrica por } 5 \text { a } 10 \\
\text { min, caminhada na } \\
\text { esteira a } 3,2 \mathrm{~km} / \mathrm{h} \\
\text { por } 15 \text { min, progres- } \\
\text { sões de acordo com } \\
\text { o limiar de dor e } \\
\text { máxima inclinação } \\
\text { na esteira de } 10 \% \\
\text { sem alterar velo- } \\
\text { cidade, por } 1 \text { ho- } \\
\text { ra, duas vezes/sem }\end{array}$ & $10 \mathrm{sem}$ & $\begin{array}{c}\text { após } 3 \\
\text { meses } \\
\text { DIC } \\
\text { DMC } \\
\text { ITB } \\
\text { após } 3 \text { anos } \\
\text { DIC } \\
\text { DMC } \\
\text { ITB }\end{array}$ & $\begin{array}{c}\uparrow 237 \% \\
\uparrow 242 \% \\
\text { não } \Delta \\
\uparrow 223 \% \\
\uparrow 188 \% \\
\text { não } \Delta\end{array}$ & $\begin{array}{c}<0,001^{\mathrm{a}} \\
<0,001^{\mathrm{a}} \\
- \\
<0,001^{\mathrm{a}} \\
<0,001^{\mathrm{a}} \\
-\end{array}$ \\
\hline \multirow[t]{2}{*}{ Lee et al. ${ }^{31}$} & 70 & $\begin{array}{l}\text { EXE } \\
(n=33)\end{array}$ & $\operatorname{sim}$ & COMBO & $\begin{array}{l}\text { Circuito ( } 2 \text { min } \\
\text { cada estação, in- } \\
\text { cluindo estações de } \\
\text { atividade aeróbia - } \\
\text { step e bicicleta } \\
\text { ergométrica e de } \\
\text { exercícios resisti- } \\
\text { dos com carga } \\
\text { leve, três vezes/ } \\
\text { sem, por } 60 \text { min }\end{array}$ & $12 \mathrm{sem}$ & $\begin{array}{l}\text { DMC } \\
\text { DIC } \\
\text { ITB } \\
\text { QV }\end{array}$ & $\begin{array}{c}\text { aumentou } \\
\text { aumentou } \\
\text { não } \Delta \\
\text { não } \Delta\end{array}$ & $\begin{array}{c}<0,05^{\mathrm{a}} \\
<0,05^{\mathrm{a}} \\
\quad- \\
\quad-\end{array}$ \\
\hline & & $\begin{array}{l}\text { CONT } \\
(\mathrm{n}=37)\end{array}$ & & & & & & & \\
\hline $\begin{array}{l}\text { Sudbrack, Sar- } \\
\text { mento-Leite }\end{array}$ & 15 & $\begin{array}{l}\text { EXE } \\
(n=15)\end{array}$ & $\operatorname{sim}$ & COMBO & $\begin{array}{l}\text { Caminhada na estei- } \\
\text { ra com velocidade } \\
\text { de } 3 \mathrm{~km} / \mathrm{h} \text {, por } 5 \mathrm{a} \\
25 \mathrm{~min}+\text { exercícios } \\
\text { resistidos com carga } \\
\text { leve }(1 \mathrm{a} 2 \mathrm{~kg})+\text { bi- } \\
\text { cicleta ergométrica } \\
\text { por } 6 \text { min com car- } \\
\text { ga de acordo com } \\
\text { tolerância individu- } \\
\text { al, três vezes } / \mathrm{sem}, \\
\text { por } 50 \text { min }\end{array}$ & $20 \mathrm{sem}$ & $\begin{array}{c}\text { DMC } \\
\text { TMC } \\
\text { QV } \\
\text { dor }\end{array}$ & $\begin{array}{c}\text { aumentou } \\
\text { aumentou } \\
\text { aumentou } \\
\text { diminuiu }\end{array}$ & $\begin{array}{l}<0,05^{\mathrm{a}} \\
<0,05^{\mathrm{a}} \\
0,001^{\mathrm{a}} \\
<0,05^{\mathrm{a}}\end{array}$ \\
\hline $\begin{array}{l}\text { Bendermacher et } \\
\text { al. }^{34}\end{array}$ & 56 & EXE & $\operatorname{sim}$ & COMBO & $\begin{array}{l}\text { Caminhada em es- } \\
\text { teira de acordo com } \\
\text { limiar de dor segui- } \\
\text { do por exercícios } \\
\text { resistidos, duas a } \\
\text { três vezes/sem, por } \\
\text { aproximadamente } \\
30 \text { min }\end{array}$ & $24 \mathrm{sem}$ & $\begin{array}{l}\text { após } 3 \\
\text { meses } \\
\text { DIC } \\
\text { DMC } \\
\text { após } 6 \\
\text { meses } \\
\text { DIC } \\
\text { DMC }\end{array}$ & $\begin{array}{l}\uparrow 187 \% \\
\uparrow 142 \% \\
\uparrow 240 \% \\
\uparrow 191 \%\end{array}$ & $\begin{array}{l}<0,0001^{\mathrm{a}} \\
<0,0001^{\mathrm{a}} \\
<0,0001^{\mathrm{a}} \\
<0,0001^{\mathrm{a}}\end{array}$ \\
\hline Adams et al. ${ }^{35}$ & 35 & $\begin{array}{l}\text { EXE } \\
(\mathrm{n}=35)\end{array}$ & $\operatorname{sim}$ & AER & $\begin{array}{l}\text { Caminhada em es- } \\
\text { teira com intensida- } \\
\text { de que induzisse } \\
\text { máxima dor da } \\
\text { claudicação em } \\
6 \text { min seguida de } \\
3 \text { min de descan- } \\
\text { so, totalizando seis } \\
\text { intervalos por ses- } \\
\text { são, três vezes/sem }\end{array}$ & $12 \mathrm{sem}$ & ER & aumentou & $0.017^{\mathrm{a}}$ \\
\hline
\end{tabular}


Tabela 1 (cont.) - Estudos sobre exercício na doença arterial obstrutiva periférica com claudicação intermitente

\begin{tabular}{|c|c|c|c|c|c|c|c|c|c|}
\hline \multirow[t]{2}{*}{ Autores } & \multirow[t]{2}{*}{$\mathrm{n}$} & \multirow[t]{2}{*}{ Grupo } & \multicolumn{4}{|c|}{ Exercício } & \multicolumn{3}{|c|}{ Resultados } \\
\hline & & & Supervisão & Modalidade & Prescrição & Duração & Variáveis & Mudança & $\mathrm{p}$ \\
\hline \multirow[t]{3}{*}{ Zwierska et al. ${ }^{32}$} & \multirow[t]{3}{*}{94} & $\begin{array}{l}\text { EXE } 1 \\
(n=30)\end{array}$ & $\operatorname{sim}$ & AER & $\begin{array}{l}\text { Exercício com } \\
\text { cicloergômetro de } \\
\text { membros superio- } \\
\text { res, } 85 \text { a } 90 \% \text { do } \\
\mathrm{VO}_{2} \text { pico, por } 2 \\
\text { min, seguido de } 1 \\
\text { min de descan- } \\
\text { so, por } 40 \text { min, } \\
\text { duas vezes/sem }\end{array}$ & $24 \mathrm{sem}$ & $\begin{array}{c}\text { G EXE } 1 \\
\text { DMC } \\
\text { DIC } \\
\mathrm{VO}_{2} \\
\text { ITB } \\
\text { NAF }\end{array}$ & $\begin{array}{c}\uparrow 51 \% \\
\uparrow 29 \% \\
\text { aumentou } \\
\text { não } \Delta \\
\text { aumentou }\end{array}$ & $\begin{array}{l}<0,001^{\mathrm{a}} \\
<0,01^{\mathrm{a}} \\
<0,01^{\mathrm{a}} \\
\quad- \\
<0,01^{\mathrm{a}}\end{array}$ \\
\hline & & $\begin{array}{l}\text { EXE } 2 \\
(n=32)\end{array}$ & $\operatorname{sim}$ & AER & $\begin{array}{l}\text { Exercício com } \\
\text { cicloergômetro de } \\
\text { membros inferio- } \\
\text { res, } 85 \text { a } 90 \% \text { do } \\
\mathrm{VO}_{2} \text { pico, por } \\
2 \mathrm{~min} \text {, seguido de } \\
1 \text { min de descan- } \\
\text { so, por } 40 \text { min, du- } \\
\text { as vezes/sem }\end{array}$ & $24 \mathrm{sem}$ & $\begin{array}{c}\text { G EXE } 2 \\
\text { DMC } \\
\text { DIC } \\
\mathrm{VO}_{2} \\
\text { ITB } \\
\text { NAF }\end{array}$ & $\begin{array}{c}\uparrow 57 \% \\
\uparrow 31 \% \\
\text { aumentou } \\
\text { não } \Delta \\
\text { aumentou }\end{array}$ & $\begin{array}{c}<0,001^{\mathrm{a}} \\
<0,01^{\mathrm{a}} \\
<0,01^{\mathrm{a}} \\
\quad- \\
<0,01^{\mathrm{a}}\end{array}$ \\
\hline & & $\begin{array}{l}\text { CONT } \\
(\mathrm{n}=32)\end{array}$ & & & & & & & \\
\hline \multirow[t]{2}{*}{ Gardner et al. ${ }^{36}$} & \multirow[t]{2}{*}{85} & $\begin{array}{l}\text { EXE 1 } \\
(\mathrm{n}=39 \\
\text { fuman- } \\
\text { tes })\end{array}$ & $\operatorname{sim}$ & AER & $\begin{array}{l}\text { Caminhada em } \\
\text { esteira de acordo } \\
\text { com limiar de } \\
\text { dor, três vezes/sem }\end{array}$ & $24 \mathrm{sem}$ & $\begin{array}{c}\text { G EXE } 1 \\
\text { DIC } \\
\text { DM } \\
\mathrm{VO}_{2} \\
\text { DTC6 } \\
\text { NAF } \\
\text { FS } \\
\text { ITB }\end{array}$ & $\begin{array}{c}\text { aumentou } \\
\text { aumentou } \\
\text { aumentou } \\
\text { aumentou } \\
\text { aumentou } \\
\text { aumentou } \\
\text { não } \Delta\end{array}$ & $\begin{array}{l}<0,05^{\text {a }} \\
<0,05^{\text {a }} \\
<0,05^{\text {a }} \\
<0,05^{\text {a }} \\
<0,05^{\text {a }} \\
<0,05^{\text {a }} \\
\quad-\end{array}$ \\
\hline & & $\begin{array}{c}\text { EXE } 2 \\
(\mathrm{n}=46, \\
\text { não fu- } \\
\text { mantes })\end{array}$ & $\operatorname{sim}$ & AER & $\begin{array}{l}\text { Caminhada em } \\
\text { esteira de acordo } \\
\text { com limiar de } \\
\text { dor, três vezes/sem }\end{array}$ & $24 \mathrm{sem}$ & $\begin{array}{c}\text { G EXE } 2 \\
\text { DIC } \\
\text { DM } \\
\mathrm{VO}_{2} \\
\text { DTC6' } \\
\text { NAF } \\
\text { FS } \\
\text { ITB }\end{array}$ & $\begin{array}{c}\text { aumentou } \\
\text { aumentou } \\
\text { aumentou } \\
\text { aumentou } \\
\text { aumentou } \\
\text { aumentou } \\
\text { não } \Delta\end{array}$ & $\begin{array}{l}<0,05^{\text {a }} \\
<0,05^{\text {a }} \\
<0,05^{\text {a }} \\
<0,05^{\text {a }} \\
<0,05^{\text {a }} \\
<0,05^{\text {a }} \\
\quad-\end{array}$ \\
\hline
\end{tabular}

${ }^{\mathrm{a}}$ Valores de p comparando pré- e pós-intervenção.

$\mathrm{AER}=$ aeróbio; $\mathrm{COMBO}=$ aeróbio + exercício resistido de baixa intensidade; $\mathrm{CONT}=$ controle; DIC = distância início claudicação; DMC = distância máxima caminhada; DPdm = duplo produto na distância máxima caminhada; DPr = duplo produto no repouso; DTC6' $^{\prime}$ distância no teste de caminhada de 6 minutos; ER = escore reabilitação (velocidade $\mathrm{x}$ inclinação no teste ergométrico); $\mathrm{EXE}=$ exercício $; \mathrm{FCe}=$ frequência cardíaca no exercício; $\mathrm{FCr}=$ frequência cardíaca no repouso; FS = fluxo sanguíneo; FSdm = fluxo sanguíneo na distância máxima caminhada; $\mathrm{FSr}=$ fluxo sanguíneo no repouso; $\mathrm{G}$ = grupo; ITB = índice tornozelo-braquial; $\mathrm{LS}=$ lactato sanguíneo; $\mathrm{NAF}=$ nível atividade física; $\mathrm{PAD}=$ pressão arterial diastólica; $\mathrm{PAS}=$ pressão artéria sistólica; $\mathrm{PCF}=$ proporção de capilares por fibra muscular; $\mathrm{QV}=$ qualidade de vida; rep = repetições; sem = semana; TCLD = tempo de caminhada livre de dor; TMC = tempo máximo caminhado; $\mathrm{VLD}$ = velocidade no limiar de dor; $\mathrm{VM}$ = velocidade máxima; $\mathrm{VO}_{2}=$ consumo máximo de oxigênio.

\section{Melhora na qualidade de vida}

A qualidade de vida após o treinamento físico melhorou significativamente em dois estudos ${ }^{25,27}$. Sudbrack e Sarmento-Leite ${ }^{25}$ avaliaram a qualidade de vida através do questionário de qualidade de vida WHOQOL e verificaram incremento no domínio físico e nas relações sociais, bem como no escore total $(p=0,001)$. Roberts et al. ${ }^{27}$ utilizaram questionário específico para portadores de doenças vasculares (Vascular Quality of Life Survey - VascuQol) e observaram aumento de $22,4 \%$ no escore total $(p<0,001)$. Já Lee et al. ${ }^{31}$, utilizando o questionário genérico de avaliação de qualidade de vida SF-36, não observaram mudança significativa no grupo tratado com exercícios, mas o grupo-controle demonstrou deterioração significativa no escore total do SF-36. 


\section{Melhora no fluxo sanguíneo}

Dois estudos avaliaram o fluxo sanguíneo no membro afetado após programa de exercício aeróbio. Ambos obtiveram aumentos significativos ${ }^{27,36}$. Roberts et al. ${ }^{27}$ mensuraram o fluxo sanguíneo através de pletismografia venosa de oclusão na perna mais sintomática (PVL 50, SciMed, Bristol, UK) antes e após teste de exercício. Gardner et al. ${ }^{36}$ avaliaram o fluxo sanguíneo do membro mais afetado sob condições de repouso, hiperemia reativa e hiperemia máxima através de pletismografia venosa de oclusão. Esses autores avaliaram os efeitos do treino aeróbio em dois grupos, fumantes e não fumantes, e obtiveram resultados semelhantes em ambos os grupos para todas variáveis; no entanto, o fluxo sanguíneo após o treinamento mostrou-se significativamente mais baixo no grupo de não fumantes na análise entre os grupos $(\mathrm{p}<0,05)$. Tal fato demonstra que o exercício aeróbio é capaz de promover melhorias também em pacientes fumantes, mas que o fluxo sanguíneo no membro afetado, menor do que o dos não fumantes, apesar de poder apresentar melhora, continuará sendo inferior ao dos pacientes não tabagistas.

Apesar dos vários efeitos benéficos demonstrados pela intervenção por exercícios, o índice tornozelo-braquial (ITB), que é o melhor preditor de risco de progressão da doença arterial periférica nos pacientes que apresentam $\mathrm{CI}^{5}$, não obteve mudança significativa em seis estur $\operatorname{dos}^{27,31-33,36-38}$ dos sete que o avaliaram. Provavelmente, não se observou melhora significativa no ITB porque o exercício incrementa o fluxo sanguíneo do membro afetado devido à melhora da circulação colateral. Isto não necessariamente afetaria a pressão sistólica das artérias tibial posterior e pediosa, pois o mecanismo que ocorre parece produzir uma redistribuição local do fluxo sanguíneo em vez de um aumento absoluto deste. Sendo assim, tal fato explicaria por que a melhora do fluxo sanguíneo no membro afetado não se traduz em mudanças significativas no $\mathrm{ITB}^{27}$. Dos estudos analisados, apenas dois eram não supervisionados $^{27,37}$, sendo que estes obtiveram resultados semelhantes quando comparados aos supervisionados. Entretanto, pesquisas sugerem que o exercício físico supervisionado leva a benefícios clinicamente mais relevantes do que o não supervisionado. No entanto, essa relevância ainda não foi demonstrada definitivamente, o que assinala a necessidade de mais estudos que objetivem incrementos na qualidade de vida de pacientes com $\mathrm{DAOP}^{23}$.

\section{Conclusão}

Na literatura pesquisada, estudos sobre a reabilitação da DAOP associada à CI através de fisioterapia ainda são escassos. Os estudos que utilizam a intervenção através de exercícios são limitados e não padronizados, o que pode contribuir para que a reabilitação não seja difundida na prática clínica. A atividade aeróbia do tipo caminhada parece ser a mais utilizada, e seus efeitos se traduzem, principalmente, na melhora do desempenho de caminhada e da qualidade de vida de portadores de CI. Além disso, esse tipo de intervenção mostra-se segura, eficaz e de baixo custo.

\section{Referências}

1. Garcia LA. Epidemiology and pathophysiology of lower extremity peripheral arterial disease. J Endovasc Ther. 2006;13(Suppl 2):3-9.

2. Medeiros AH, Chalegre ST, Carvalho CC. Eletroestimulação muscular: alternativa de tratamento coadjuvante para pacientes com doença arterial obstrutiva periférica. J Vasc Bras. 2007;6:156-62.

3. Pinto DM, Mandil A. Claudicação intermitente: do tratamento clínico ao intervencionista. Rev Bras Cardiol Invas. 2005;13:261-9.

4. Robless P, Mikhailidis DP, Stansby GP. Cilostazol for peripheral arterial disease. Cochrane Database Syst Rev. 2007;1:CD003748.

5. Norgren L, Hiatt WR, Dormandy JA, et al. Inter-Society Consensus for the Management of Peripheral Arterial Disease (TASC II). J Vasc Surg. 2007;45 Suppl S:S5-S67.

6. Parry DJ, Grant PJ, Scott DJ. Atherothrombotic risk factor clustering in healthy male relatives of male patients with intermittent claudication. J Vasc Surg. 2004;40:891-8.

7. Makdisse M, Ramos LR, Moreira F, et al. Escore para rastrear idosos (= 75 anos) de alto risco para doença arterial periférica. Arq Bras Cardiol. 2007;88:630-6.

8. Yoshida RA, Matida CK, Sobreira ML, et al. Estudo comparativo da evolução e sobrevida de pacientes com claudicação intermitente, com ou sem limitação para exercícios, acompanhados em ambulatório específico. J Vasc Bras. 2008;7:112-22.

9. Chi YW, Jaff MR. Optimal risk factor modification and medical management of the patient with peripheral arterial disease. Catheter Cardiovasc Interv. 2008;71:475-89.

10. Gardner AW, Montgomery PS, Parker DE. Physical activity is a predictor of all-cause mortality in patients with intermittent claudication. J Vasc Surg. 2008;47:117-22.

11. Durazzo AE, Sitrângulo CD, Presti C, Silva ES, Luccia N. Doença arterial obstrutiva periférica: que atenção temos dispensado à abordagem clínica dos pacientes? J Vasc Bras. 2005;4:255-64.

12. Rice TW, Lumsden AB. Optimal medical management of peripheral arterial disease. Vasc Endovascular Surg. 2006;40:312-27.

13. Schaan B, Mandelli NC. Conduta na doença arterial periférica em pacientes diabéticos. Rev Soc Cardiol RS. 2004;XIII. 
http://sociedades.cardiol.br/sbc-rs/revista/2004/02/artigo02. pdf. Acessado: 25.09.2008.

14. Aronow WS. Management of peripheral arterial disease of the lower extremities. Compr Ther. 2007;33:247-56.

15. Hankey GJ, Norman PE, Eikelboom JW. Medical treatment of peripheral arterial disease. JAMA. 2006;295:547-53.

16. Neto SS, Nascimento JL. Doença arterial obstrutiva periférica: novas perspectivas de fatores de risco. Rev Para Med. 2007;21:35-9.

17. Garg PK, Tian L, Criqui MH, Liu K, Ferrucci L, Guralnik JM. Physical activity during daily life and mortality in patients with peripheral arterial disease. Circulation. 2006;114:242-8.

18. Askew CD, Green S, Walker PJ, et al. Skeletal muscle phenotype is associated with exercise tolerance in patients with peripheral arterial disease. J Vasc Surg. 2005;41:802-7.

19. Ambrosetti M, Salerno M, Boni S, Daniele G, Tramarin R, Pedretti RF. Economic evaluation of a short-course intensive rehabilitation program in patients with intermittent claudication. Int Angiol. 2004;23:108-13.

20. Crowther RG, Spinks WL, Leicht AS, Sangla K, Quigley F, Golledge J. Effects of a long-term exercise program on lower limb mobility, physiological responses, walking performance, and physical activity levels in patients with peripheral arterial disease. J Vasc Surg. 2008;47:303-9.

21. Wind J, Koelemay MJ. Exercise therapy and the additional effect of supervision on exercise therapy in patients with intermittent claudication. Systematic review of randomised controlled trials. Eur J Vasc Endovasc Surg. 2007;34:1-9.

22. Hodges LD, Sandercock GR, Das SK, Brodie DA. Randomized controlled trial of supervised exercise to evaluate changes in cardiac function in patients with peripheral atherosclerotic disease. Clin Physiol Funct Imaging. 2008;28:32-7.

23. Bendermacher BL, Willigendael EM, Teijink JA, Prins MH. Supervised exercise therapy versus non-supervised exercise therapy for intermittent claudication. Cochrane Database Syst Rev. 2006;2:CD005263.

24. Murphy TP, Hirsch AT, Ricotta JJ, et al. CLEVER Steering Committee. The claudication: exercise vs. endoluminal revascularization (CLEVER) study: rationale and methods. J Vasc Surg. 2008;47:1356-63.

25. Sudbrack AC, Sarmento-Leite R. Efetividade do exercício na claudicação. Rev Bras Cardiol Invas. 2007;15:261-6.

26. Andreozzi GM, Leone A, Laudani R, Deinite G, Martini R. Acute impairment of the endothelial function by maximal treadmill exercise in patients with intermittent claudication, and its improvement after supervised physical training. Int Angiol. 2007;26:12-7.

27. Roberts AJ, Roberts EB, Sykes K, De Cossart L, Edwards P, Cotterrell D. Physiological and functional impact of an unsupervised but supported exercise programme for claudicants. Eur J Vasc Endovasc Surg. 2008;36:319-24.

28. Treesak C, Kasemsup V, Treat-Jacobson D, Nyman JA, Hirsch AT. Cost-effectiveness of exercise training to improve claudication symptoms in patients with peripheral arterial disease. Vasc Med. 2004;9:279-85.
29. Wolosker N, Munia MA, Rosoky R, et al. Tratamento cirúrgico para claudicação intermitente em pacientes que não melhoram com o tratamento clínico. Arq Bras Cardiol. 2004;82:445-9.

30. Aquarius AE, Denollet J, Hamming JF, Breek JC, De Vries J. Impaired health status and invasive treatment in peripheral arterial disease: a prospective 1-year follow-up study. J Vasc Surg. 2005;41:436-42.

31. Lee HL, Mehta T, Ray B, Heng MS, McCollum PT, Chetter IC. A non-randomised controlled trial of the clinical and cost effectiveness of a supervised exercise programme for claudication. Eur J Vasc Endovasc Surg. 2007;33:202-7.

32. Zwierska I, Walker RD, Choksy SA, Male JS, Pockley AG, Saxton JM. Upper-vs lower-limb aerobic exercise rehabilitation in patients with symptomatic peripheral arterial disease: a randomized controlled trial. J Vasc Surg. 2005;42:1122-30.

33. Wang J, Zhou S, Bronks R, Graham J, Myers S. Effects of supervised treadmill walking training on calf muscle capillarization in patients with intermittent claudication. Angiology. 2009;60:36-41.

34. Bendermacher BL, Willigendael EM, Nicolaï SP, et al. Supervised exercise therapy for intermittent claudication in a community-based setting is as effective as clinic-based. J Vasc Surg. 2007;45:1192-6.

35. Adams J, Ogola G, Stafford P, Koutras P, Hartman J. Highintensity interval training for intermittent claudication in a vascular rehabilitation program. J Vasc Nurs. 2006;24:46-9.

36. Gardner AW, Killewich Montgomery PS, Katzel LI. Response to exercise rehabilitation in smoking and nonsmoking patients with intermittent claudication. J Vasc Surg. 2004;39:531-8.

37. Manfredini F, Malagoni AM, Mascoli F, et al. Training rather than walking the test in-train out program for homebased rehabilitation in peripheral arteriopathy. Circ J. 2008;72:946-52.

38. Ratliff DA, Puttick M, Libertiny G, Hicks RC, Earby LE, Richard T. Supervised exercise training for intermittent claudication: lasting benefit at three years. Eur J Vasc Endovasc Surg. 2007;34:322-6.

39. Câmara LC, Santarém JM, Wolosker N, Dias RM. Exercícios resistidos terapêuticos para indivíduos com doença arterial obstrutiva periférica: evidências para a prescrição. J Vasc Bras. 2007;6:246-56.

40. Leng GC, Fowler B, Ernst E. Exercise for intermittent claudication. Cochrane Database Syst Rev. 2000;2:CD000990.

41. Rosoky RM, Wolosker N, Puech-Leao P. Performance of patients with intermittent claudication undergoing physical training, with or without an aggravation of arterial disease: retrospective cohort study. Clinics. 2006;61:535-8.

\footnotetext{
Correspondência:

Camila Pereira Leguisamo

Rua Capitão Eleutério 69, 304, Centro

CEP 99010-060 - Passo Fundo, RS

Tel.: (54) 9124.0216, (54) 3314.6473

Fax: (54) 3316.8380

E-mail: camila@upf.br
} 